\title{
Neuronal Acetylcholine Receptors That Bind $\alpha$-Bungarotoxin Mediate Neurite Retraction in a Calcium-Dependent Manner
}

\author{
Phyllis C. Pugh and Darwin K. Berg \\ Department of Biology, University of California at San Diego, La Jolla, California 92093-0357
}

Neuronal membrane components that bind $\alpha$-bungarotoxin with high affinity have only recently been shown unambiguously to function as nicotinic receptors. Activation of the receptors increases intracellular levels of free calcium in neurons. In the chick ciliary ganglion, where the receptors have been studied in some detail, they have been shown to have a predominantly nonsynaptic location on neurons and may be concentrated on pseudodendrites emerging from the somata. This has raised questions about the physiological significance of the receptors for the neurons. Here we show that activation of the receptors on isolated ciliary ganglion neurons in cell culture produces neurite retraction. Focal application of either nicotine or $\mathrm{ACh}$ at low concentrations induces the retraction, and $\alpha$-bungarotoxin blocks the effect. The retraction requires external calcium and is confined to the individual neurite stimulated with agonist. Brief exposure to elevated concentrations of $\mathrm{K}^{+}$also induces neurite retraction, and both the $\mathrm{K}^{+}$-induced and the nicotine-induced retractions can be prevented by the calcium channel blocker $\omega$-conotoxin. The results suggest that activation of the $\alpha$-bungarotoxin-binding nicotinic receptors on neurites triggers activation of voltage-gated calcium channels presumably by depolarizing the membrane, and that together they permit sufficient calcium to enter the neurite to prevent further outgrowth and induce retraction.

[Key words: nicotinic receptors, neuronal ACh receptors, $\alpha$-bungarotoxin, neurite extension, growth cones, intracellular calcium, ciliary ganglion neurons]

Nicotinic ACh receptors (AChRs) are abundant in the nervous system and comprise a family of receptor subtypes (for review, see Sargent, 1993). Speculations about their likely physiological roles have been heavily influenced by the much-studied muscle AChR. Located in the postsynaptic membrane at the neuromuscular junction, muscle AChRs function as cation-selective ligand-gated ion channels responsible for fast, excitatory synaptic input from the motor nerve terminal. Numerous examples of nicotinic responses have been observed in neurons as well.

\footnotetext{
Received Mar. 4, 1993; revised June 2, 1993; accepted July 20, 1993.

We thank Dr. M. H. Benn (University of Calgary) for his kind gift of MLA, Lynn Ogden for expert technical assistance, and Susan Schoonmakcr, Leticia Oliva, Teri Yuen, Everardo Gutierrez, and Michael Silverberg for preparation of ciliary ganglion cell cultures. Grant support was provided by the National Institutes of Health (Grants R01 NS12601 and P01 NS25916) and the California Tobacco Related-Disease Research Program.

Correspondence should be addressed to Darwin K. Berg, Department of Biology, 0357; University of California at San Diego, 9500 Gilman Drive, La Jolla, CA 92093-0357.

Copyright (C) 1994 Society for Neuroscience $0270-6474 / 94 / 140889-08 \$ 05.00 / 0$
}

Only at preganglionic neuron synapses on autonomic neurons and at motoneuron synapses on Renshaw cells, however, is there good evidence that neuronal AChRs mediate fast, excitatory synaptic transmission. This has led to speculation that some neuronal AChR subtypes may serve other functions such as that of modulating neuronal signaling or regulating aspects of neuronal development.

One class of neuronal AChRs that has spawned numerous questions about function is the species that binds the snake neurotoxin $\alpha$-bungarotoxin ( $\alpha \mathrm{Bgt}$ ). Numerous attempts over a number of years failed to demonstrate a function for such receptors ( $\alpha$ Bgt-AChRs). New impetus for the analysis of $\alpha \mathrm{Bgt}-$ AChRs was provided by the cloning of the AChR $\alpha 7$ gene. Not only was the $\alpha 7$ gene product shown to be a subunit of at least some $\alpha$ Bgt-AChRs (Schoepfer et al., 1990; Keyser et al., 1993), but it was also found to produce functional ligand-gated ion channels, apparently as a homomer, when expressed in Xenopus oocyles (Couturier el al., 1990; Seguela et al., 1993). Subsequent experiments have provided evidence that native $\alpha \mathrm{Bgt}-\mathrm{AChRs}$ on neurons do function as ligand-gated ion channels (Alkondon et al., 1992; Zorumski et al., 1992; Z.-w. Zhang and D. K. Berg, unpublished observations). Previous failures to detect an electrophysiological response for the receptors probably resulted from their rapid rate of agonist-induced desensitization and from their being over shadowed by the responses of other AChR subtypes on the neurons.

The physiological role of $\alpha \mathrm{Bgt}-\mathrm{AChRs}$ in vivo remains unresolved. In the chick ciliary ganglion, where $\alpha \mathrm{Bgt}-\mathrm{AChRs}$ have been studied in some detail, the receptors are located predominantly in nonsynaptic membrane on the neurons both in the embryo and in the adult (Jacob and Berg, 1983; Loring et al., 1985). Few, if any, $\alpha$ Bgt-AChRs are concentrated in the postsynaptic membrane, making it unlikely that the receptors contribute directly to synaptic transmission. Instead, other AChRs, distinguished by their ability to bind the monoclonal antibody $\mathrm{mAb} 35$ and their inability to bind $\alpha \mathrm{Bgt}$, are primarily found in the postsynaptic membrane and appear to be responsible for nicotinic transmission through the ganglion (Jacob et al., 1984; Loring et al., 1984; Loring and Zigmond, 1987). These largely synaptic receptors are referred to here as mAb 35-AChRs and, as a population, contain the $\alpha 3, \beta 4$, and $\alpha 5$ gene products (Vernallis et al., 1993). They do not contain detectable amounts of the $\alpha 7$ gene product. In contrast, $\alpha \mathrm{Bgt}$-AChRs on the neurons contain the $\alpha 7$ gene product but not the $\alpha 3, \beta 4$, or $\alpha 5$ gene products, indicating that $\alpha \mathrm{Bgt}-\mathrm{AChRs}$ and $\mathrm{mAb} 35-\mathrm{AChRs}$ are clearly distinct receptor species. Ciliary ganglion $\alpha \mathrm{Bgt}$-AChRs also lack the $\alpha 8$ gene product (Vernallis et al., 1993) found in some forms of brain and retina $\alpha \mathrm{Bgt}-\mathrm{AChRs}$ (Schoepfer et al., 1990; Keyser et al., 1993). 
Recently it has been shown that activation of $\alpha$ Bgt-AChRs on chick ciliary ganglion neurons can produce a marked elevation in the level of intracellular free calcium (Vijayaraghavan et al., 1992). Receptors made by expressing the chick and rat $\alpha 7$ genes in Xenopus oocytes are highly permeable to calcium (Galzi et al., 1992; Seguela et al., 1993). If the same is true of native $\alpha \mathrm{Bgt}-\mathrm{AChRs}$, the receptors would have the potential of influencing a vast array of calcium-related events in the neurons.

One function that may be regulated by $\alpha \mathrm{Bgt}-\mathrm{AChRs}$ is neurite extension. Numerous studies have indicated that conditions which change intracellular calcium levels in the growth cone can dramatically alter the growth pattern of the neurite (for review, see Kater and Mills, 1991). Nicotinic antagonists have been shown to enhance the number and mean length of neurites from retinal ganglion cells in culture, implicating nicotinic receptors in the regulation of neurite outgrowth (Lipton et al., 1988). In the case of the pheochromocytoma cell line PCI2, $\alpha \mathrm{Bgt}$ has been shown to promote the extension of neurites (Quik et al., 1990). Since PC12 cells can synthesize and release ACh under some conditions (Greene and Rein, 1977), the $\alpha$ Bgt effects may result from its serving as an $\mathrm{AChR}$ antagonist.

Electron microscopic studies with HRP-conjugated $\alpha \mathrm{Bgt}$ suggest that $\alpha$ Bgt-AChRs on chick ciliary ganglion neurons are concentrated preferentially on pseudodendrites extending from the somata (Jacob and Berg, 1983). Given the location of the receptors and their ability to elevate intracellular levels of free calcium, it seemed possible that $\alpha \mathrm{Bgt}-\mathrm{AChRs}$ could regulate neuronal morphology. We report here that activation of $\alpha \mathrm{Bgt}-$ AChRs on ciliary ganglion neurons in dissociated cell culture inhibits neurite extension and induces a partial retraction of the processes in a calcium-dependent manner. Further, experiments with calcium channel blockers suggest that the retraction depends at least in part on functional voltage-gated calcium channels, presumably recruited by activation of the $\alpha$ Bgt-AChRs.

\section{Materials and Methods}

Ciliary ganglion cultures. Freshly dissected ciliary ganglia from $8 \mathrm{~d}$ chick embryos were dissociated with trypsin as previously described (Nishi and Berg, 1981) and plated on $35 \mathrm{~mm}$ dishes coated either with $0.5 \mathrm{mg}$ of poly-D-lysine alone or with poly-D-lysine plus $15 \mathrm{mg}$ of laminin. The culture medium consisted of Eagle's minimal essential medium supplemented with $10 \%(\mathrm{v} / \mathrm{v})$ fetal bovine serum and $3 \%(\mathrm{v} / \mathrm{v})$ embryonic chick eye extract (Nishi and Berg, 1981). Cell cultures were maintained overnight (14-30 hr) in a humidified incubator with $5 \% \mathrm{CO}_{2}, 95 \%$ air at $37^{\circ} \mathrm{C}$ before being taken for analysis.

Neurite measurements. For measurement of neurite length, cultures were perfused at $37^{\circ} \mathrm{C}$ with culture medium containing $10 \mathrm{~mm}$ HEPES,

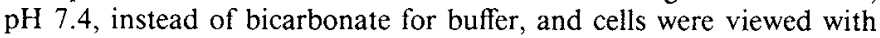
phase-contrast optics at either $320 \times$ or $400 \times$ magnification. Neurite lengths were quantified using a reticulated eyepiece to measure the distance from the origin of the neurite at the soma perimeter to the most distal edge of the growth cone, defined as either the outer edge of the lamellapodia or the farthest reaching filopodium. Only data from neurons having an unbranched neurite were used for the compilations. Photomicrographs were taken with Kodak T-max 100 film at $400 \times$ with phase-contrast optics using a Leitz Diavert microscope.

Stimulation paradigms. AChR agonists in perfusion medium (vehicle) were applied to neurites by pressure from micropipettes positioned 5 $15 \mu \mathrm{m}$ from the growing tip of the neurite. The protocol usually consisted of three $10 \mathrm{sec}$ pulses $6 \mathrm{~min}$ apart $\left(3 \times 10^{\prime \prime}, 6^{\prime}\right)$. In some cases the protocol was changed to deliver five $2 \mathrm{sec}$ pulses of agonist 3 min apart $\left(5 \times 2^{\prime \prime}\right.$, $3^{\prime}$ ). For long-term nicotine application, the initial culture volume was reduced to $0.5 \mathrm{ml}$ and the cells were perfused with $1 \mu \mathrm{M}$ nicotine at a rate of at least $2.5 \mathrm{ml} / \mathrm{min}$ for $3 \mathrm{~min}$ and then $0.17 \mathrm{ml} / \mathrm{min}$ for the remainder of the $72 \mathrm{~min}$ observation time. Agonists included nicotine alone or $\mathrm{ACh}$ in the presence of atropine. When $\mathrm{K}^{+}$was used to stimulate, it was substituted for nicotine in the micropipette (final concen- tration of $25 \mathrm{~mm}$ ); the stimulation protocol involved three 10 sec pulses $6 \mathrm{~min}$ apart. Neurites were monitored at regular intervals for approximately $1 \mathrm{hr}$ after applying agonist or until complete retraction had occurred and no regrowth was observed for at least $5 \mathrm{~min}$.

When AChR antagonists were employed, they were present in the perfusion medium prior to and during tests with agonist, but were not present in the micropipette with the nicoline. $\alpha$ Bgt was incubated with the cells at $50 \mathrm{~nm}$ for at least $60 \mathrm{~min}$ before perfusing the cells at $10 \mathrm{~nm}$ and stimulating with agonist. Methyllycaconitine (MLA) was present at $50 \mathrm{~nm}$ both before (at least $15 \mathrm{~min}$ ) and during the tests with nicotine. $d$-Tubocurarine (dTC) was present at $0.1 \mathrm{~mm}$ both before (at least 10 min) and during the tests. When $\mathrm{ACh}$ was substituted for nicotine as agonist, atropine was added to the perfusion medium at $0.25 \mu \mathrm{M} 5 \mathrm{~min}$ before and during the test to block activation of muscarinic receptors and was present in the micropipette as well. The minus $\mathrm{Ca}^{2+}$ condition was obtained by including $1 \mathrm{~mm}$ EDTA and $10 \mathrm{~mm}$ BAPTA in the micropipette with the nicotine. The calcium channel blockers $\omega$-conotoxin $(\omega-\mathrm{Ctx} ; 2 \mu \mathrm{M})$ and nifedipine $(10 \mu \mathrm{M})$, when present, were added to the perfusion medium for 30 and $15 \mathrm{~min}$, respectively, prior to testing.

Statistics. Statistics were computed using STATVIEw $512+$ (BrainPower, Inc.). Significance values were determined using a three-way ANOVA, including a negative control (vehicle), a positive control (agonist), and the experimental condition to be compared. In some cases a two-tailed $t$ test was applied instead of the ANOVA, as indicated.

Materials. White Leghorn chick embryos were obtained locally and maintained at $37^{\circ} \mathrm{C}$ in a humidified incubator. Tissue extracts and culture media were prepared as described previously (Nishi and Berg, 1981). $\alpha$ Bgt was purified from Bungarus multicinctus venom (Ravdin and Berg, 1979). MLA was the kind gift of Dr. M. H. Benn (University of Calgary). Eagle's minimal essential medium powder was purchased from GIBCO; fetal bovine serum, from Whittaker; $\mathrm{ACh}$, atropine, and $\omega$-conotoxin GVIA, from Research Biochemicals Inc.; laminin from Upstate Biotechnology Inc.; and poly-D-lysine, nicotine, dTC, nifedipine, EDTA, HEPES, and BAPTA, from Sigma.

\section{Results}

Neurite extension and growth cone morphology in culture. Ciliary ganglion neurons from $8 \mathrm{~d}$ chick embryos were used to test the effects of $\alpha$ Bgt-AChRs on neurites in cell culture. The neurons are known to have the receptors at this time (Smith et al., 1985) and can readily survive and extend processes in culture (Helfand et al., 1976; Nishi and Berg, 1981). The neurons were plated at low density and examined after relatively short periods of time (14-30 hr) so that cells would remain isolated from each other for the duration of the experiment. Usually polylysine was used as the substratum to facilitate cellular adhesion to the surface while permitting easy visualization of growth cones.

Many neurons extended one or more neurites ranging in length from 10 to over $200 \mu \mathrm{m}$. Growth cones exhibited a range of morphologies. The simplest represented neurite tips with no apparent filopodia or lamellapodia. The most complex had multiple filopodia and lamellapodia covering approximately 100 $\mu \mathrm{m}^{2}$. In some cases, a single filopodium extended up to $20 \mu \mathrm{m}$ beyond the end of the neurite trunk.

To determine the rate of neurite growth or retraction, neurite lengths were measured at various times over a $72 \mathrm{~min}$ period. Neurite length was taken to be the curvilinear distance between the point of neurite origin at the cell soma and the point of farthest extension whether that represented the distal edge of the lamellapodia or the end of a filopodium. Filopodia appeared to occur with equal frequency throughout the time period under the test conditions employed. Excluding them from the measurements, that is, taking the distalmost extension of the neurite as being the outer edge of the lamellapodia, did not change the values calculated for neurite extension or retraction in the experiments described below.

Nicotine-induced retraction of neurites. Studies with rhoda- 


\begin{tabular}{|c|c|c|c|}
\hline Condition & $n$ & $\begin{array}{l}\text { Relative } \\
\text { neurite } \\
\text { length }(\%)\end{array}$ & $\begin{array}{l}\text { Change in } \\
\text { length } \\
(\mu \mathrm{m})\end{array}$ \\
\hline Control & 18 & $116 \pm 7+\dagger$ & $10 \pm 3$ \\
\hline Vehicle & 15 & $122 \pm 8+\dagger$ & $12 \pm 4$ \\
\hline Vehicle $\left(5 \times 2^{\prime \prime}, 3^{\prime}\right)$ & 8 & $115 \pm 6 \dagger$ & $9 \pm 3$ \\
\hline \multicolumn{4}{|l|}{ Nicotine } \\
\hline $0.5 \mu \mathrm{M}$ & 10 & $121 \pm 10 \dagger \dagger$ & $9 \pm 3$ \\
\hline $1 \mu \mathrm{M}$ & 50 & $66 \pm 5^{*}$ & $-13 \pm 2$ \\
\hline $2 \mu \mathrm{M}$ & 14 & $88 \pm 5^{*} \dagger$ & $-7 \pm 2$ \\
\hline $10 \mu \mathrm{M}$ & 14 & $92 \pm 5^{*}+$ & $-3 \pm 2$ \\
\hline $1 \mu \mathrm{M}\left(5 \times 2^{\prime \prime}, 3^{\prime}\right)$ & 25 & $75 \pm 11^{*}$ & $-13 \pm 5$ \\
\hline $1 \mu \mathrm{M}$ (long-term) & 25 & $113 \pm 6+\dagger$ & $4 \pm 2$ \\
\hline $1 \mu \mathrm{M}(+10 \mu \mathrm{M}$ leak $)$ & 11 & $121 \pm 9+\dagger$ & $9 \pm 3$ \\
\hline \multicolumn{4}{|l|}{ Nicotine $(1 \mu \mathrm{M})+$ blockers } \\
\hline$\alpha \operatorname{Bgt}(50 \rightarrow 10 \mathrm{~nm})$ & 21 & $103 \pm 4 \dagger \dagger$ & $2 \pm 3$ \\
\hline MLA (50 nм) & 13 & $105 \pm 6+\dagger$ & $3 \pm 3$ \\
\hline $\operatorname{dTC}(100 \mu \mathrm{M})\left(5 \times 2^{\prime \prime}, 3^{\prime}\right)$ & 11 & $115 \pm 2 \dagger \dagger$ & $9 \pm 2$ \\
\hline \multicolumn{4}{|l|}{ Nicotine $(10 \mu \mathrm{M})+\alpha$ Bgt } \\
\hline$(50 \rightarrow 10 \mathrm{~nm})$ & 14 & $118 \pm 6 \ddagger$ & $9 \pm 3$ \\
\hline Vehicle $+\alpha$ Bgt $(50 \rightarrow 10 \mathrm{~nm})$ & 13 & $109 \pm 3 \dagger$ & $6 \pm 2$ \\
\hline
\end{tabular}

Individual neurites were treated with the indicated agents and scored for retraction either as a percentage change in length or as an absolute difference in length as described in Figure 3. Values represent the mean \pm SEM for the number of neurites indicated as $n$. The stimulation paradigm involved three $10-\mathrm{sec}$ pulses of vehicle with the indicated concentrations of agonist at $6 \mathrm{~min}$ intervals, except in the cases designated $5 \times 2^{\prime \prime}, 3^{\prime}$ where agonist was delivered in five 2 -sec pulses at $3 \mathrm{~min}$ intervals, and in the case designated "long-term" where the nicotine was present throughout the stimulation $(12 \mathrm{~min})$ and observation $(60 \mathrm{~min})$ periods. In one condition $[1 \mu \mathrm{M}(+10 \mu \mathrm{M}$ leak)] a second micropipette containing $10 \mu \mathrm{M}$ nicotine was positioned near the growth cone for the duration of the experiment but was not used for stimulation. Statistics were computed for the relative neurite lengths, comparing the values to those obtained both with the corresponding vehicle alone and with $1 \mu \mathrm{M}$ nicotine, except for the condition with $10 \mu \mathrm{M}$ nicotine $+\alpha \mathrm{Bgt}$ which was compared with vehicle alone and $10 \mu \mathrm{M}$ nicotine alone.

* Significantly different from vehicle $(p<0.01)$.

$\dagger$ Significantly different from $1 \mu \mathrm{M}$ nicotine $(p<0.05)$.

† Significantly different from $1 \mu \mathrm{M}$ nicotine $(p<0.01)$.

$\ddagger$ Significantly different from $10 \mu \mathrm{M}$ nicotine $(p<0.01)$.

mine-labeled $\alpha$ Bgt have previously shown that chick ciliary ganglion neurons in culture have $\alpha$ Bgt-AChRs on their neurites as well as on their cell bodies (Ravdin et al., 1981). To determine whether AChRs might influence neurite extension, nicotinic agonists were applied directly to individual growth cones by pressure from a nearby micropipette. Stimulation paradigms usually involved applying agonist for three $10 \mathrm{sec}$ periods $6 \mathrm{~min}$ apart. The discontinuous pattern of application was chosen to reduce possible desensitization of $\alpha \mathrm{Bgt}$-AChRs on the growth cones. The physical act of applying solution from the micropipette did not alter the basal rate of neurite growth. The mean rate of neurite extension on a polylysine substratum in control cultures was $7.2 \pm 2.9 \mu \mathrm{m} / \mathrm{hr}$ (mean $\pm \mathrm{SEM}, n=18$ ), resulting in an average increase of about $20 \%$ during a $1 \mathrm{hr}$ period of observation. Neurites receiving vehicle alone in the standard application paradigm displayed the same mean rate of extension and similar net elongation during the test period (Table 1).

Neurites receiving $1 \mu \mathrm{M}$ nicotine from the micropipette underwent a mean net retraction of approximately $35 \%$ in length during the $1 \mathrm{hr}$ period of observation (Table 1). The extent of retraction varied considerably among neurites, ranging from complete retraction in about one-seventh of the cases to no
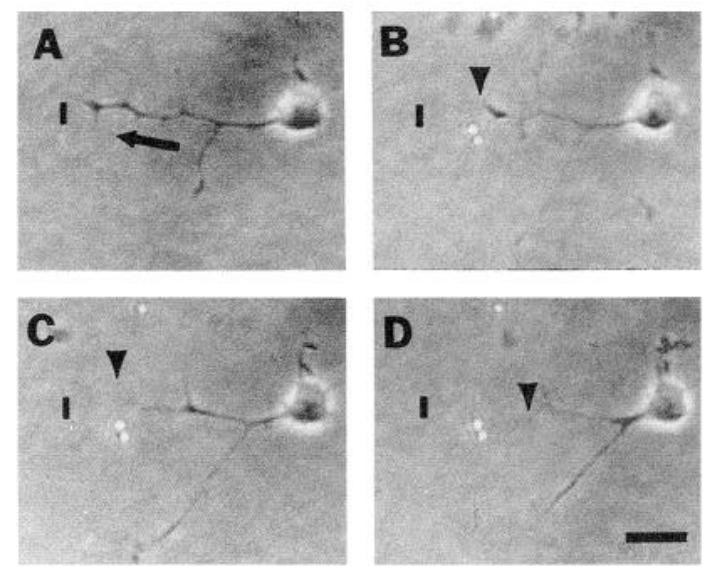

Figure 1. Neurite undergoing nicotine-induced retraction. Photomicrographs were taken with phase-contrast optics of the cell and its neurites before $(A), 2 \mathrm{~min}$ after $(B), 35 \mathrm{~min}$ after $(C)$, and $62 \mathrm{~min}$ after $(D)$ stimulation with $1 \mu \mathrm{M}$ nicotine for three $10 \mathrm{sec}$ pulses $6 \mathrm{~min}$ apart. The large arrow in $A$ indicates the position of the micropipette containing nicotine; the vertical bars indicate the maximal extension of the neurite at the beginning of stimulation, and the arrowheads indicate the end of the neurite at the indicated times. The stimulated branch retracted $43 \%$ during the observation period, decreasing from an initial length of about $70 \mu \mathrm{m}$ to a final length of about $40 \mu \mathrm{m}$. The unstimulated (vertical) branch remained essentially unchanged in length. Scale bar, $20 \mu \mathrm{m}$.

retraction or net outgrowth in about a tenth of the cases. The time course of retraction also varied greatly but usually reached a maximum within $45 \mathrm{~min}$. In some cases, complete retraction occurred almost immediately upon exposure to nicotine. In the other extreme, some neurites underwent a partial retraction followed by resumption of growth at the control rate after about $50 \mathrm{~min}$.

An example of an intermediate case (the most common) of nicotine-induced neurite retraction is shown in Figure 1, where photomicrographs taken at regular intervals during the observation period record the retraction of a growth cone and neurite. The neurite was bifurcated at the outset, and agonist was applied only to the tip of the horizontal branch. Over a $62 \mathrm{~min}$ observation period the horizontal branch receded $30 \mu \mathrm{m}$ while the vertical branch remained essentially unchanged. In addition to illustrating the effects of nicotine, the example implies that the retraction may be a local event, being restricted to the neurite or neuritic branch that is stimulated. This was confirmed for unbranched neurites by studying neurons having multiple processes. Out of 15 such cases where the stimulated neurite retracted, only 2 of 17 unstimulated neurites extending from the same neurons did so. The frequency of retraction for the unstimulated neurites was indistinguishable from that described above for control neurites or neurites receiving vehicle alone.

Tests were usually confined to neurons with unbranched neurites. Examples of results obtained from neurites treated either with vehicle alone or with vehicle plus nicotine are shown in Figure 2, where changes in neurite length are plotted as a function of time. For neurites receiving only vehicle (Fig. $2 A$ ), the examples have been chosen to illustrate the most common result (triangles) along with two of the more extreme cases (circles and squares). For neurons receiving nicotine, three of the most common responses are shown (Fig. $2 B$ ) as well as three of the more extreme responses (Fig. 2C). Figure 3 compiles the results from all vehicle- and nicotine-treated neurites as histograms. Each 

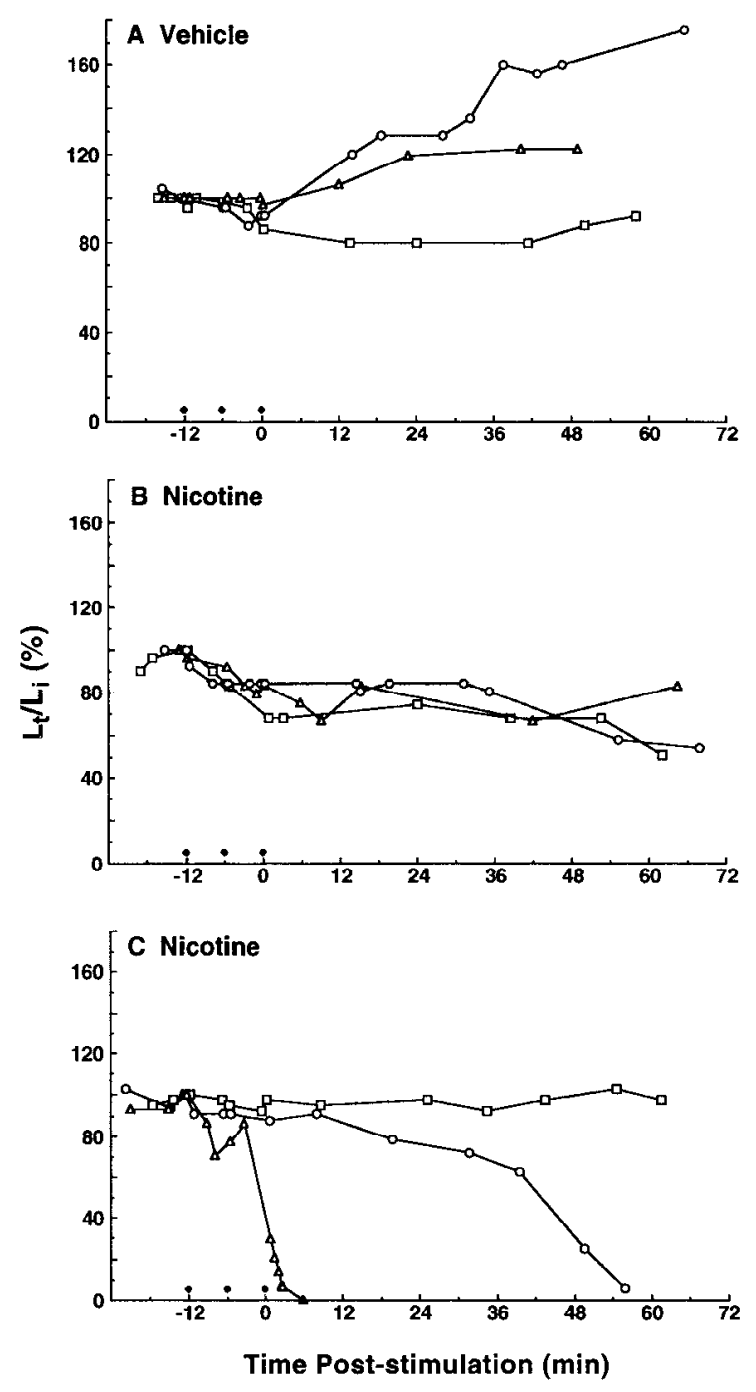

Figure 2. Time course of neurite retraction. Neurites were treated either with vehicle $(A)$ or with vehicle containing nicotine $(B, C)$ as described in Figure $I$ and then measured for net length at the indicated times. For each time point, the neurite length $\left(L_{t}\right)$ is expressed as a percentage of the initial length $\left(L_{i}\right)$ at the beginning of the stimulation period (-12 $\mathrm{min})$. Three examples of vehicle-treated neurites were chosen $(A)$ to illustrate the most common result (triangles) as well as the rare extremes of extensive growth (circles) and no growth (squares). Three examples of nicotine-treated neurites were chosen $(B)$ to illustrate the most common time course and extent of neurite retraction (circles, triangles, and squares), while three others were chosen $(C)$ to illustrate the less common behaviors of complete and abrupt retraction (triangles), gradual retraction followed by sharp and complete retraction later in the test period (circles), and no retraction (squares). The three solid diamonds in each panel indicate the timing of vehicle or nicotine treatment.

entry represents the relative change observed for a neurite during the test period, calculated by comparing its length at the point of maximum sustained retraction with its length at the beginning of the test period. A sustained retraction was taken to be one lasting at least $5 \mathrm{~min}$. When neurite length changed continuously during the test period as in the case of vehicle-treated neurites and some agonist-treated neurites, the relative change was calculated by comparing the final length at the end of the test period
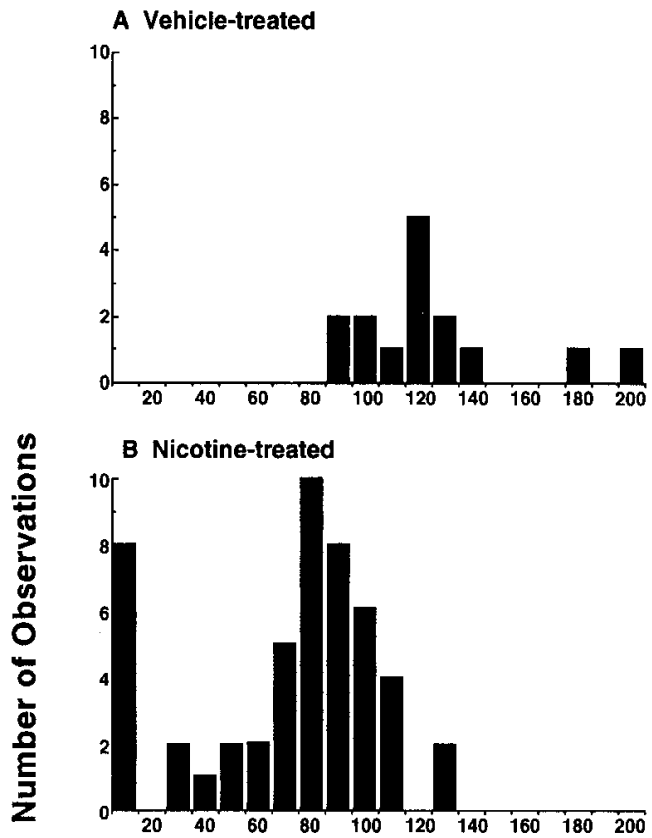

C $\alpha$ Bgt-pretreated

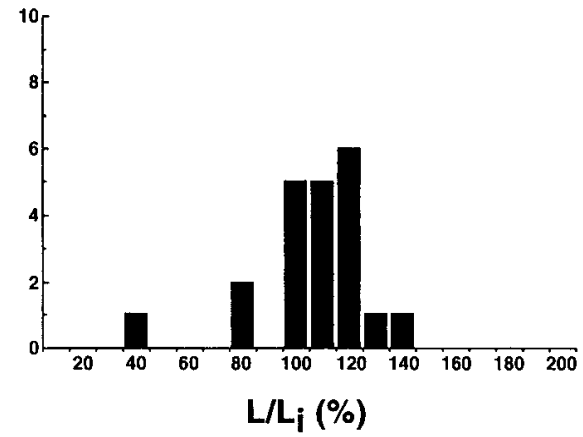

Figure 3. Histogram of neurite lengths following treatments to induce retraction. Neurites were stimulated as in Figure 1 with vehicle alone $(A)$, vehicle plus nicotine $(B)$, or vehicle plus nicotine after blockade with $\alpha$ Bgt (C). The relative change in length for each neurite was calculated by expressing the length at the sustained maximum retraction $(L)$ as a percentage of the initial length $\left(L_{i}\right)$ at the beginning of stimulation. When neurite length continually changed during the test period and did not undergo a sustained retraction, the relative length was calculated by expressing the final length as a percentage of the initial length (see Results). The values have been pooled into bins of $10 \%$; for example, the $20 \%$ bin included all neurites that retracted to $11-20 \%$ of their initial lengths. The results show that nicotine treatment shifts the distribution of neurite lengths to shorter values and that $\alpha \mathrm{Bgt}$ treatment blocks the effect.

to the starting length. The point of maximum sustained retraction was used whenever possible, however, because some nicotine-treated neurites retracted but subsequently recovered partially and reinitiated growth as described above. The overall rcsults are the same whether final lengths or maximum retracted lengths are used: those test conditions yielding values significantly different from controls do so by either method of scoring.

Varying the concentration of nicotine in the micropipette indicated that $1 \mu \mathrm{M}$ usually produced the maximum effect. Decreasing the concentration to $0.5 \mu \mathrm{M}$ had no effect on neurite growth, and increasing the concentration to 2 or $10 \mu \mathrm{M}$ caused 
less retraction than did $1 \mu \mathrm{M}$ (Table 1). Changing the stimulation paradigm did not significantly enhance the effect. Applying the nicotine in five $2 \mathrm{sec}$ pulses $3 \mathrm{~min}$ apart instead of the usual three $10 \mathrm{sec}$ pulses $6 \mathrm{~min}$ apart did not increase the effect (Table 1). Extending the number and time period of nicotine pulses (e.g., six $10 \mathrm{sec}$ pulses at 3 min intervals or thirteen $2 \mathrm{sec}$ pulses at 1 min intervals) also failed to increase the extent of retraction (data not shown).

The results suggest competing processes that produce a narrow window of effective agonist concentrations. High concentrations of agonist may partially desensitize the receptors while insufficient concentrations of agonist may not reach a required threshold. Evidence that a threshold exists and that it may vary among experiments comes from the observation that for some sets of cultures the standard paradigm of challenging with nicotine at $1 \mu \mathrm{M}$ produced little effect while $2 \mu \mathrm{M}$ nicotine induced the usual retraction (data not shown). (This necessitated positive controls for nicotine-induced retraction being routinely conducted for all experimental conditions.) Desensitization also influences the outcome. Continuous application of $1 \mu \mathrm{M}$ nicotine for $72 \mathrm{~min}$ did not induce neurite retraction (Table 1). Moreover, when high concentrations of agonist are present in the micropipettc, steady lcakage between stimulation pulses apparently desensitizes the receptors. This was demonstrated by showing that the standard stimulation paradigm with $1 \mu \mathrm{M}$ nicotine was unsuccessful at inducing retraction when a second micropipette containing $10 \mu \mathrm{M}$ nicotine was positioned near the growth cone but not used for stimulation (Table 1). Desensitization produced by such leakage probably accounts for the observation that 10 $\mu \mathrm{M}$ nicoline produces less retraction in the standard stimulation paradigm than does $1 \mu \mathrm{M}$.

The ability of nicotine to induce neurite retraction was not confined to cells on a substratum of poly-D-lysine. Similar results were obtained when the dishes were coated with laminin in addition to polylysine. The mean neurite length prior to nicotine application was longer for neurons grown on laminin/poly-Dlysine ( $99 \pm 11 \mu \mathrm{m}$, mean $\pm \mathrm{SEM}, n=21)$ than on poly-Dlysine alone ( $54 \pm 2 \mu \mathrm{m}, n=350 ; p<0.0001, t$-test). Nonetheless, application of $1 \mu \mathrm{m}$ nicotine in the standard protocol (three $10 \mathrm{sec}$ pulses, $6 \mathrm{~min}$ apart) readily blocked neurite extension. By the end of the $1 \mathrm{hr}$ observation period, the mean length of nicotine-treated neurites was $108 \pm 5 \%$ (mean \pm SEM, $n=12$ ) of their initial length while that of control neurites receiving vehicle alone was $168 \pm 35 \%(n=9)$ of their initial length. Thus, on a substratum of laminin/poly-D-lysine the nicotine-treated neurites were, on average, a third shorter than vehicle-treated neurites by the end of the test period.

$\alpha$ Bgt-AChRs mediate nicotinic effects on neurites. The contribution of $\alpha$ Bgt-AChRs to nicotine-induced neurite retraction was tested by incubating cells with $50 \mathrm{nM} \alpha$ Bgt and then perfusing with $10 \mathrm{nM}$. The toxin is specific in this concentration range, inhibiting the response of $\alpha$ Bgt-AChRs while not affecting mAb 35-AChRs on the neurons (Ravdin and Berg, 1979; Vijayaraghavan et al., 1992). $\alpha$ Bgt blocked the effects of both 1 and $10 \mu \mathrm{M}$ nicotine on neurite growth (Table 1). A second nicotinic antagonist with high affinity for $\alpha$ Bgt-AChRs is MLA. At $50 \mathrm{~nm}$ the ligand should occupy most of the $\alpha \mathrm{Bgt}$-AChRs on ciliary ganglion neurons while binding to very few mAb 35AChRs (Vijayaraghavan et al., 1992). As with $\alpha$ Bgt, MLA blocked the effects of nicotine on neurite outgrowth. dTC, a nicotinic antagonist not expected to discriminate between $\alpha \mathrm{Bgt}-\mathrm{AChRs}$
Table 2. Summary of neurite treatments with $\mathrm{ACh} \pm \alpha \mathrm{Bgt}$

\begin{tabular}{ccc} 
ACh $(\mu \mathrm{M})$ & Agonist alone & Agonist $+\alpha$ Bgt \\
\hline 50 & $96 \pm 8(25)^{*}$ & $130 \pm 6(12) \dagger$ \\
100 & $88 \pm 4(20)^{*}$ & $95 \pm 10(14)^{*}$ \\
200 & $94 \pm 8(9)^{*}$ & $95 \pm 9(9)^{*}$ \\
1000 & $120 \pm 11(6)$ & ND \\
\hline
\end{tabular}

Individual neurites were stimulated with the indicated concentration of $\mathrm{ACh}$ in the standard stimulation paradigm $\pm \alpha \mathrm{Bgt}$ and were scored as percentage of initial length as described in Table 1. Atropine at $0.25 \mu \mathrm{M}$ was included in the perfusion and all ACh solutions. Values represent the mean \pm SEM for the number of determinations indicaled in parentheses. ND, Not determined.

* Significantly different from that obtained with vehicle alone (Table $1 ; p<0.05$ ). $\dagger$ Significantly different from that obtained with the same concentration of agonist in the absence of $\alpha \mathrm{Bgt}(p<0.01)$.

and $\mathrm{mAb} 35-\mathrm{AChRs}$, produced a slightly greater nominal blockade than did either $\alpha$ Bgt or MLA, but the three antagonists were not significantly different in their effects either from each other or from that observed with vehicle alone (Table 1). $\alpha$ Bgt by itself did not significantly affect neurite outgrowth (Table 1).

The presumed endogenous agonist for nicotinic receptors is $\mathrm{ACh}$. To determine whether $\mathrm{ACh}$ induced neurite retraction and whether $\alpha$ Bgt could block it, neurites were stimulated with several concentrations of $\mathrm{ACh}$ in the presence and absence of toxin. At $50 \mu \mathrm{M}$ ACh produced a substantial retraction, and $\alpha$ Bgt blocked it (Table 2). At higher concentrations (100 and $200 \mu \mathrm{M}$ ) the agonist also achieved retraction, but mAb 35-AChRs appeared to be responsible since $\alpha$ Bgt did not block the effects. At $1 \mathrm{~mm}$ ACh did not alter neurite growth, probably because leakage of agonist from the micropipette desensitized the receptors as described above for nicotine applications. Atropine at $0.25 \mu \mathrm{M}$ was routinely included in the micropipette along with the $\mathrm{ACh}$ to eliminate muscarinic receptors as possible contributors to any effects observed.

The most likely mechanism by which activation of $\alpha$ BgtAChRs influences neurite outgrowth is by changing the level of intracellular free calcium. It is known that the elevation of intracellular calcium caused by $\alpha$ Bgt-AChRs depends on extracellular calcium (Vijayaraghavan et al., 1992). Accordingly, the role of extracellular calcium in the present experiments was examined by adding calcium chelators to the calcium normally present in the micropipette used to apply nicotine. This approach minimized the exposure of the neurite to calcium-free conditions but ensured that little, if any, extraccllular calcium would be available during the times in which the $\alpha$ Bgt-AChRs were activated. Application of $1 \mu \mathrm{M}$ nicotine by the standard protocol (three $10 \mathrm{sec}$ pulses at $6 \mathrm{~min}$ intervals) in the presence of the calcium chelators was unable to inhibit neurite growth. The relative neurite length at the end of the test period was 125 $\pm 9 \%$ of the initial length (mean \pm SEM, $n=13 ; p<0.000001$ from $1 \mu \mathrm{M}$ nicotine). The value was indistinguishable from that obtained for unstimulated neurites or neurites challenged only with vehicle (Table 1).

Involvement of voltage-dependent $\mathrm{Ca}^{2+}$ channels. Recent results confirm that $\alpha$ Bgt-AChRs function as ligand-gated ion channels and cause a rapid, transient depolarization of chick ciliary ganglion neurons (Zhang and Berg, unpublished observations). Accordingly, the ability of $\alpha \mathrm{Bgt}$-AChRs to elevate intracellular calcium in the neurons may result either from the 


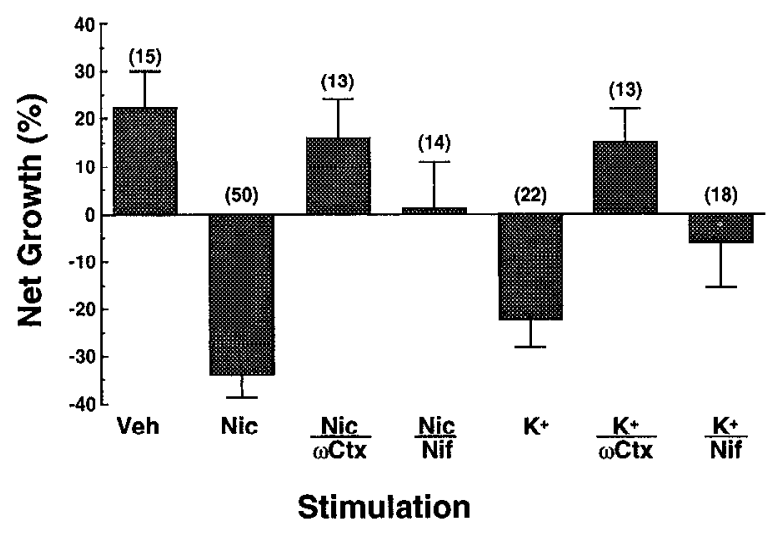

Figure 4. Effects of voltage-gated calcium channel blockers on nicotine-induced neurite retraction. Neurites were stimulated as in Figure 1 either with nicotine or with $25 \mathrm{mM} \mathrm{K}^{+}$in the presence or absence of the indicated calcium channel blockers. The results were calculated as described in Figure 3 to obtain a mean \pm SEM in each case and are shown as the net percentage difference from the starting length. The number of neurites analyzed is shown in parentheses. Veh, vehicle; Nic, nicotine; $K^{+}$, potassium; $\omega C t x$, $\omega$-conotoxin; Nif, nifedipine. Both nicotine and $\mathrm{K}^{+}$treatments resulted in neurite lengths that were significantly different from the vehicle control $(p<0.001)$ and not different from each other. Both $\omega$-Ctx and nifedipine significantly protected against the nicotine effect, yielding values for neurite length that were significantly different from the nicotine-treated cases $(p<0.01)$ while not being significantly different from vehicle controls.

receptors being highly permeable to calcium or from their being able to activate voltage-gated calcium channels in the neurites. To investigate the contribution of voltage-dependent calcium channels to neurite retraction in the cultures, neurites were stimulated with elevated $\mathrm{K}^{+}$concentrations $(25 \mathrm{~mm})$ from the $\mathrm{mi}-$ cropipette. The brief pulses of $\mathrm{K}^{+}$should depolarize the membrane and activate voltage-gated channels if present. Using the standard stimulation protocol described above for nicotine, the elevated concentration of $\mathrm{K}^{+}$induced a retraction comparable to that caused by nicotine (Fig. 4).

Evidence that the $\mathrm{K}^{+}$-induced retraction of neurites involved activation of voltage-gated calcium channels was obtained through the use of the calcium channel blockers. $\omega$-Ctx is thought to block $\mathrm{N}$-type calcium channels, while nifedipine is thought to block primarily L-type channels (for reviews, see Bean, 1989; Tsien and Tsien, 1990). $\omega$-Ctx at $2 \mu \mathrm{M}$ prevented the $\mathrm{K}^{+}$treatment from inducing neurite retraction. The mean neurite length at the end of the observation period was $115 \pm 7 \%(n=12)$, a value not significantly different from that for vehicle-treated neurites. Nifedipine produced a less complete reversal of the $\mathrm{K}^{+}$effect, resulting in a mean neurite length of $93 \pm 8 \%(n=$ 18) at the end of the test period.

Both calcium channel blockers also reversed the effects of nicotine on neurite length (Fig. 4). Thus, in the presence of 2 $\mu \mathrm{M} \omega-\mathrm{Ctx}$, neurites treated with nicotine achieved a mean final length of $116 \pm 8 \%(n=13)$ at the end of the test period, which was not significantly different from that seen for vehicle-treated neurites (Fig. 4). Similarly, incubation in $10 \mu \mathrm{M}$ nifedipine allowed neurites treated with nicotine to reach a mean final length of $101 \pm 19 \%(n=14)$ by the end of the test period. If the two calcium channel blockers are specific under these conditions, their ability to prevent nicotine from inducing neurite retraction in culture indicates that $\alpha \mathrm{Bgt}-\mathrm{AChRs}$ achieve their effects on neurites at least in part by activating voltage-gated calcium channels.

\section{Discussion}

The major findings reported here are that activation of $\alpha$ BgtAChRs can induce neurite retraction and that the retraction is likely to result from calcium influx. The conclusion that $\alpha \mathrm{Bgt}$ AChRs are responsible for much of the nicotinic effect is supported by the pharmacological evidence showing that low concentrations of $\alpha \mathrm{Bgt}$ and MLA can each block most of the nicotine-induced neurite retraction. At the concentrations used both the $\alpha \mathrm{Bgt}$ and MLA would have occupied essentially all of the $\alpha$ Bgt-AChRs while binding to few, if any, mAb 35-AChRs.

Previous studies have shown that increasing the level of intraccllular frec calcium can retard growth cone motility and induce neurite retraction (Kater and Mills, 1991). A similar mechanism is likely to account for the nicotine-induced neurite retraction observed here since removal of extracellular calcium blocked the effect. $\alpha$ Bgt-AChRs when activated on ciliary ganglion neurons are known to elevate intracellular levels of free calcium (Vijayaraghavan et al., 1992). Some of the calcium increase may result from ion flux directly through the receptor channel since neuronal AChRs are, in general, permeable to calcium (Mulle et al., 1992; Vernino et al., 1992). Receptors created by expression of the $\alpha 7$ gene product in Xenopus oocytes have a particularly high permeability to calcium (Seguela et al., 1993), and chick ciliary ganglion $\alpha \mathrm{Bgt}-\mathrm{AChRs}$ are known to contain $\alpha 7$ gene product (Vernallis et al., 1993).

Voltage-gated calcium channels represent a second pathway by which extracellular calcium could enter the cells. During embryonic development, ciliary ganglion neurons have both $\mathrm{N}$ and L-type calcium channels on their presynaptic nerve terminals (Gray et al., 1990, 1992). Since activation of ciliary ganglion $\alpha \mathrm{Bgt}-\mathrm{AChRs}$ can produce a rapid depolarization of the membrane as indicated above, the depolarization may activate voltage-gated calcium channels and thereby augment calcium entry. Evidence that voltage-gated calcium channels can trigger neurite retraction in the present studies is provided by the observation that brief applications of concentrated $\mathrm{K}^{+}$are sufficient to mimic nicotine in reversing neurite outgrowth. Moreover, $\omega$-Ctx blocked the effects of $\mathrm{K}^{+}$, implicating $\mathrm{N}$-type calcium channels in the process. The fact that $\omega$-Ctx was also able to block nicotine-induced neurite retraction provides strong evidence that $\alpha \mathrm{Bgt}$-AChRs achieve their effects on the neurites in large part by activating voltage-gated channels to augment calcium influx.

Additional evidence for involvement of voltage-gated channels is provided by the finding that nifedipine can block nicotineinduced neurite retraction. Inferences about the participation of voltage-gated calcium channels, however, must be considered tentative because they depend heavily on the specificity of the calcium channel blockers. Preliminary results indicate that $\omega$-Ctx does not block the current response resulting from activation of $\alpha \mathrm{Bgt}-\mathrm{AChRs}$ on the ccll soma, but the results are less clear with nifedipine (Zhang and Berg, unpublished observations). An additional complication is that conceivably $\alpha$ Bgt-AChRs on growth cones differ in some pharmacological manner from $\alpha \mathrm{Bgt}$ AChRs on the cell body, thereby limiting the value of characterizing the latter population to learn about the former. Other approaches will be needed to obtain a definitive answer about 
the role of voltage-gated channels in neurite retraction mediated by $\alpha$ Bgt-AChRs.

The narrow range of nicotine concentrations capable of inducing neurite retraction probably reflects a combination of factors. A "threshold effect" may be imposed by voltage-gated channels if their participation is required for neurite retraction. Activation of $\alpha$ Bgt-AChRs that was insufficient to depolarize the membrane to threshold for activating the voltage-gated channels would have little effect. Activation of more than enough receptors to reach threshold may or may not induce additional retraction over that observed at threshold, depending on the calcium dependence of the process. A second consideration, operative at high agonist concentrations, is receptor desensitization. AChRs produced by expressing the $\alpha 7$ gene product in Xenopus oocytes have a very rapid rate of agonist-induced desensitization (Couturier et al., 1990). Ciliary ganglion $\alpha$ BgtAChRs may behave similarly, explaining why short pulses of agonist spaced several minutes apart were effective at inducing retraction while chronic exposure was not. As the double pipette experiments showed, even leakage of agonist from the micropipette between pulses is apparently sufficient to desensitize the receptors when a high concentration of agonist is used. Leakageinduced desensitization of the receptors, therefore, can account for the finding that a smaller effect was observed with high concentrations of agonist (e.g., $10 \mu \mathrm{M}$ nicotine, $1 \mathrm{~mm} \mathrm{ACh}$ ) than with low concentrations (e.g., $1 \mu \mathrm{M}$ nicotine, $50 \mu \mathrm{M} \mathrm{ACh}$ ). The fact that $\alpha 7$-containing AChRs not only desensitize rapidly but also prefer nicotine over ACh (Couturier et al., 1990; Vernallis et al., 1993; Zhang and Berg, unpublished observations) may explain why $\alpha \mathrm{Bgt}$ was unable to block the neurite retractions elicited by intermediate concentrations of ACh (100, $200 \mu \mathrm{M})$. Under these conditions the $\alpha \mathrm{Bgt}-\mathrm{AChRs}$ were probably desensitized but sufficient $\mathrm{mAb} 35-\mathrm{AChRs}$ apparently remained active to produce the response.

Variation observed in the time course and extent of neurite retraction following application of nicotine could arise from differences in the number and distribution of $\alpha \mathrm{Bgt}-\mathrm{AChRs}$ or voltage-gated ion channels among neurites. Other sources of variability may have to do with differences in the effectiveness of calcium buffering among neurites or in the efficiency of intracellular molecular mechanisms mediating calcium-dependent neurite collapse. The mechanisms have yet to be fully elucidated but are known to include calcium regulation of actin filament and/or microtubule assembly (Kater and Mills, 1991). Including filopodia in the length measurements did not enhance the variability among experiments. Filopodia seemed to occur with cqual probability under all test conditions; cxcluding them from the measurements did not alter any of the conclusions. Reports of filopodia collapse in previous experiments may have depended on long-term elevation of intracellular free calcium (Kater and Mills, 1991) rather than the transient increases likely to have been achieved with the protocols used here.

Previous reports have indicated that cholinergic antagonists in the case of chick retinal cells (Lipton et al., 1988) and $\alpha \mathrm{Bgt}$ in the case of rat PC12 cells (Quik et al., 1990) can increase neurite length. Since both types of cultures can make and release $\mathrm{ACh}$ under appropriate conditions (Greene and Rein, 1977; Lipton, 1988), the effects can be interpreted as relieving an ongoing ACh-induced inhibition of neurite outgrowth. Indeed, the concentration of endogenous $\mathrm{ACh}$ in the culture medium of chick retinal cultures was estimated to be $0.5 \mu \mathrm{M}$ (Lipton, 1988). No significant effect of $\alpha \mathrm{Bgt}$ alone was observed on neurite length in the present studies. The likely reason is that extracellular $\mathrm{ACh}$ concentrations in the cultures used here were too low to inhibit neurite outgrowth. The cultures were plated at a low cell density and were taken at early times so that isolated neurites from a single cell could be examined without interference from ncurites of other cclls. Though ciliary ganglion neurons can release $\mathrm{ACh}$ from the tips of growing neurites (Hume et al., 1983), the sparseness of neurites in the present case plus the presence of cholinesterases in the culture medium make it highly improbable that significant amounts of ACh could have accumulated in the medium during the test period. The absence of an $\alpha \mathrm{Bgt}$ effect on unstimulated neurons supports this contention.

Fluorescence studies with rhodamine-labeled $\alpha$ Bgt have shown that $\alpha \mathrm{Bgt}-\mathrm{AChRs}$ are present on the neurites as well as the somata of ciliary ganglion neurons in cell culture (Ravdin et al., 1981). It is not clear, however, whether the receptors responsible for inducing neurite retraction are confined to the neurites and growth cones. Though the micropipette was positioned to optimize application of agonist to the growth cone, some agonist could have reached the soma as well. A second path that might have triggered a somatic response involves the voltage-gated channels. Activation of voltage-gated channels by $\alpha \mathrm{Bgt}-\mathrm{AChRs}$ even distally on the neurites might have elicited action potentials that invaded the soma and promoted substantial calcium entry throughout the cell. An argument against the soma being the critical site at which calcium entry triggers neurite retraction is that application of nicotine to a single neurite did not necessarily induce collapse of other neurites emanating from the same cell.

One function for $\alpha$ Bgt-AChRs in vivo suggested by the present results is a remodeling of dendritic morphology in the ganglion. By embryonic day 7 ciliary neurons in the ganglion have extended fine dendritic-like processes referred to as pseudodendrites and have received functional synaptic contacts from preganglionic terminals (Landmesser and Pilar, 1972, 1978). By embryonic day 10 the dendritic processes have retracted to allow the preganglionic terminals to form large calyces cupping the axon hillock region of the neurons. $\alpha \mathrm{Bgt}-\mathrm{AChRs}$ are present in the ganglion by embryonic day 7 and increase in number up through hatching (Chiappinelli and Giacobini, 1978). Histochemical studies at the ultrastructural level with HRP-labeled $\alpha \mathrm{Bgt}$ indicate that the receptors may be most concentrated on the pseudodendrites (Jacob and Berg, 1983). It is possible that ACh released even in a diffuse manner from the preganglionic terminals may serve to activate $\alpha \mathrm{Bgt}-\mathrm{AChRs}$ distally, inducing a collapse of the pseudodendrite back into the soma. Additional experiments will be required to determine whether growing neurites are influenced by $\alpha \mathrm{Bgt}-\mathrm{AChR}$ activation in vivo where the neurons must contend with a three-dimensional matrix and substrata much more complex than presented here in cell culture.

The fact that large numbers of $\alpha$ Bgt-AChRs remain on the neurons well into adulthood invites speculation about other possible functions. $\alpha \mathrm{Bgt}-\mathrm{AChRs}$ on the cell soma may participate in a kind of nonsynaptic signaling, responding to $\mathrm{ACh}$ released either from other neuronal somata in the ganglion or from preganglionic terminals at nonsynaptic sites. Some activation of $\alpha$ Bgt-AChRs may also occur by diffusion of transmitter 
away from synaptic sites during periods of intense synaptic activity. The purposes of such signaling, however, can only be hypothesized at present.

A final speculation offered here is that $\alpha \mathrm{Bgt}-\mathrm{AChRs}$ may have functions not dictated by a somal location. Substantial numbers of the receptors may be transported down the axon to serve as presynaptic receptors at nerve-muscle junctions in the periphery. (No effort was made in the present study to distinguish axons from dendrites in cell culture.) During development presynaptic $\alpha$ Bgt-AChRs could provide a mechanism for terminating axonal growth at locations where the axon acquired the ability to release transmitter. In the adult the receptors might provide positive feedback for transmitter release. Activation of the receptors by $A C h$ released either from the same terminal or from adjacent terminals would further elevate intracellular levels of calcium and thereby enhance transmitter release. The prevalence and physiological significance of presynaptic AChRs are subjects likely to attract increasing attention.

\section{References}

Alkondon M, Pereira EFR, Wonnacott S, Albuquerque EX (1992) Blockade of nicotinic currents in hippocampal neurons defines methyllycaconitine as a potent and specific receptor antagonist. Mol Pharmacol 41:802-808.

Bean BP (1989) Classes of calcium channels in vertebrate cells. Annu Rev Physiol 51:367-384.

Chiappinelli VA, Giacobini E (1978) Time course of appearance of $\alpha$-bungarotoxin binding sites during development of chick ciliary ganglion and iris. Neurochem Res 3:465-478.

Couturier S, Bertrand D, Matter J-M, Hernandez M-C, Bertrand S, Millar N, Valera S, Barkas T, Ballivet M (1990) A neuronal nicotinic acetylcholine receptor subunit $(\alpha 7)$ is developmentally regulated and forms a homo-oligomeric channel blocked by $\alpha$-BTX. Neuron 5:847856.

Galzi J-L, Devillers-Thiery A, Hussy N, Bertrand S, Changeux J-P, Bertrand D (1992) Mutations in the channel domain of a neuronal nicotinic receptor convert ion selectivity from cationic to anionic. Nature 359:500-505.

Gray DB, Bruses JL, Pilar GR (1992) Developmental switch in the pharmacology of $\mathrm{Ca}^{2+}$ channels coupled to acetylcholine release. Neuron 8:715-724.

Gray DB, Zelazny D, Manthay N, Pilar G (1990) Endogenous modulation of $\mathrm{ACh}$ release by somatostatin and the differential roles of $\mathrm{Ca}^{2+}$ channels. J Neurosci 10:2687-2698.

Greene LA, Rein G (1977) Synthesis, storage and release of acetylcholine by a noradrenergic pheochromocytoma cell line. Nature 268: 349-351.

Helfand SL, Smith GA, Wessells NK (1976) Survival and development in culture of dissociated parasympathetic neurons from ciliary ganglia. Dev Biol 50:541-547.

Hume RI, Role LW, Fischbach GD (1983) Acetylcholine release from growth cones detected with patches of acetylcholine receptor-rich membranes. Nature 305:632-634.

Jacob MH, Berg DK (1983) The ultrastructural localization of $\alpha$-bungarotoxin binding sites in relation to synapses on chick ciliary ganglion neurons. J Neurosci 3:260-271.

Jacob MH, Bcrg DK, Lindstrom JM (1984) Shared antigenic determinant between the Electrophorus acetylcholine receptor and a synaptic component on chicken ciliary ganglion neurons. Proc Natl Acad Sci USA 81:3223-3227.

Kater SB, Mills LR (1991) Regulation of growth cone behavior by calcium. J Neurosci 11:891-899.

Keyser KT, Britto LRG, Schoepfer R, Whiting P, Cooper J, Conroy W,
Brozozowska-Prechtl A, Karten HJ, Lindstrom J (1993) Three subtypes of $\alpha$-bungarotoxin-sensitive nicotinic acetylcholine receptors are expressed in chick retina. J Neurosci 13:442-454.

Landmesser L, Pilar G (1972) The onset and development of transmission in the chick ciliary ganglion. J Physiol (Lond) 222:691-713.

Landmesser L, Pilar G (1978) Interactions between neurons and their targets during in vivo synaptogenesis. Fed Proc 37:2016-2022.

Lipton SA (1988) Spontaneous release of acetylcholine affects the physiological nicotinic responses of rat retinal ganglion cells in culture. J Neurosci 8:3857-3868.

Lipton SA, Frosch MP, Phillips MD, Tauck DL, Aizenman E (1988) Nicotinic antagonists enhance process outgrowth by rat retinal ganglion cells in culture. Science 239:1293-1296.

Loring RH, Zigmond RE (1987) Ultrastructural distribution of ${ }^{125} \mathrm{I}-$ toxin $F$ binding sites on chick ciliary neurons: synaptic localization of a toxin that blocks ganglionic nicotinic receptors. J Neurosci 7:21532162.

Loring RH, Chiappinelli VA, Zigmond RE, Cohen JB (1984) Characterization of a snake venom neurotoxin which blocks nicotinic transmission in the avian ciliary ganglion. Neuroscience 11:989-999.

Loring RH, Dahm LM, Zigmond RE (1985) Localization of $\alpha$-bungarotoxin binding sites in the ciliary ganglion of the embryonic chick: an autoradiographic study at the light and electron microscopic level. Neuroscience 14:645-660.

Mulle C, Choquet D, Korn H, Changeux J-P (1992) Calcium influx through nicotinic receptor in rat central neurons: its relevance to cellular regulation. Neuron 8:135-143.

Nishi R, Berg DK (1981) Two components from eye tissue that differentially stimulate growth and development of ciliary ganglion neurons in cell culture. J Neurosci 1:505-513.

Quik M, Cohen R, Audhya T, Goldstein G (1990) Thymopoietin interacts at the $\alpha$-bungarotoxin site of and induces process formation in PC12 pheochromocytoma cells. Neuroscience 39:139-150.

Ravdin PM, Berg DK (1979) Inhibition of neuronal acetylcholine sensitivity by $\alpha$-toxins from Bungarus multicinctus venom. Proc Natl Acad Sci USA 76:2072-2076.

Ravdin PM, Nitkin RM, Berg DK (1981) Internalization of $\alpha$-bungarotoxin on neurons induced by a neurotoxin that blocks neuronal acetylcholine sensitivity. J Neurosci 1:849-861.

Sargent PB (1993) The diversity of neuronal nicotinic acetylcholine receptors. Annu Rev Neurosci 16:403-443.

Schoepfer R, Conroy WG, Whiting P, Gore M, Lindstrom J (1990) Brain $\alpha$-bungarotoxin binding protein cDNAs and MAbs reveal subtypes of this branch of the ligand-gated ion channel gene superfamily. Neuron 5:35-48.

Seguela P, Wadiche J, Dineley-Miller K, Dani JA, Patrick JW (1993) Molecular cloning, functional properties, and distribution of rat brain $\alpha 7$ : a nicotinic cation channel highly permeable to calcium. J Neurosci 13:596-604.

Smith MA, Stollberg J, Lindstrom JM, Berg DK (1985) Characterization of a component in chick ciliary ganglia that cross-reacts with monoclonal antibodies to muscle and electric organ acetylcholine reccptor. J Ncurosci 5:2726-2731.

Tsien RW, Tsien RY (1990) Calcium channels, stores, and oscillations. Annu Rev Cell Biol 6:715-760.

Vernallis AB, Conroy WG, Berg DK (1993) Neurons assemble acetylcholine receptors with as many as three kinds of subunits and can segregate subunits among receptor subtypes. Neuron 10:451-464.

Vernino S, Amador M, Luetje CW, Patrick J, Dani JA (1992) Calcium modulation and high calcium permeability of neuronal nicotinic acetylcholine receptors. Neuron 8:127-134.

Vijayaraghavan S, Pugh PC, Zhang Z-w, Rathouz MM, Berg DK (1992) Nicotinic receptors that bind $\alpha$-bungarotoxin on neurons raise intracellular free $\mathrm{Ca}^{2+}$. Neuron 8:353-362.

Zorumski CF, Thio LL, Isenberg KE, Clifford DB (1992) Nicotinic acetylcholine currents in cultured postnatal rat hippocampal neurons. Mol Pharmacol 41:931-936. 